DOE/ ER/ $45477-1$

\title{
EMBEDDED MICROCLUSTERS IN ZEOLITES AND CLUSTER BEAM SPUTTERING .. SIMULATION ON PARALLEL COMPUTERS
}

Progress Report

September 1992 - September 1993

Priya Vashishta, Rajiv K. Kalia and Donald L. Greenwell Concurrent Computing Laboratory for Materials Simulations Physics Department Louisiana State University Baton Rouge, Louisiana 7(0803-40(01

September 1993

PREPARED FOR THE U.S. DEPARTMENT OF ENERGY UNDER GRANT NUMBER DE-FG05-92ER 45477 
TABLE OF CONTENTS

TABLE OF CONTENTS 1

EXECUTIVE SUMMARY 2

RESEARCH ACCOMPLISHMENTS 3

$\$ 1$ Multi-million Particle Molecular-Dynamics

Simulations on Parallel Machines

$\$ 2$ Structural Transformation in Microporous Silica 5

$\$ 3$ Novel high-Pressure Phase of Amorphous Silica 6

$\$ 4$ Structural and Dynamical Correlations in ORIENTATIONALly Disordered SOlid C60

\$5 Parallel Quantum Dynamics Simulations 7

$\$ 6$ WORK IN PROGRESS

$\$ 7$ DOE Supported Publications, Invited Talks at Major CONFERENCES, AND ABSTRACTS 


\title{
ANNUAL PROGRESS REPORT (September 15, 1992 - September 14, 1993)
}

PROPOSAL TITLE: Embedded Microclusters in Zeolites and Cluster Beam Sputtering Simulation on Parallel Computers

\section{GRANT NUMBER: DE-FG()5-92ER45477}

\section{PRINCIPAL}

INVESTIGATORS: Priya Vashishta, Rajiv K. Kalia, and Donald Greenwell

\author{
INSTITUTION: Concurrent Computing Laboratory for Materials Simulations \\ Louisiana State University, Baton Rouge, LA 70803-4()(01 \\ Phone: (504) 388-1342; Fax: (504) 388-5855 \\ E-mail: priyav@bit.csc.Isu.edu
}

\section{EXECUTIVE SUMMARY}

An optimal time-space multi-resolution approach has been designed to carry out large-scale molecular-dynamics (MD) simulations on distributed-memory MIMD (Multiple Instructions Multiple Data) machines. This approach involves highly efficient algorithms for long-range Coulomb forces and three-body interactions. Tests on Intel's 512-node Touchstone Delta machine at Caltech indicate that an MD time step for a covalent material with 4.2 million atoms takes only 5 seconds! This is the most efficient parallel implementation of the MD approach for real mater ' 11 s.

The multi-resolution MD approach has been used to investigate structural correlations in porous silica - an environmentally safe thermal insulator used in refrigeration, in passive solar energy collection devices, as a solid-state catalyst, and as an embedding material in optical switches. The important structural parameters such as the internal surface area and surface-to-volume ratio of pores, pore size distribution, fractal dimension, and the mean particle size have been calculated over a wide range of densities. The simulation results are in accordance with structural measurements.

Molecular-dynamics simulations have also been performed to provide a microscopic understanding of recent pioneering high-pressure experiments on silica glasses at the National Synchrotron Light Source at Brookhaven National Laboratory. The simulations reveal a structural transition to a new high-pressure amorphous phase with corner- and edge-sharing $\mathrm{SiO}_{6}$ octahedra.

Parallel algorithms have also been designed for an $a b$ initio quantum dynamics approach to materials simulations. The implementation on MasPar's 8,192-node SIMD (Single Instruction Multiple Data) machine achieves $64 \%$ of the peak performance. With this approach, we have investigated the nature of electron transport in materials. We have also developed a tight-binding MD approach to investigate the influence of orientational disorder on structural correlations and phonon spectra of $\mathrm{C}_{60}$ solid. The results are in excellent agreement with neutron-scattering measurements.

Currently, large-scale MD simulations ( $10^{6}$ atoms) are being performed to investigate the relationship between structure, dynamics, and mechanical properties and the influence of environment, composition, and stress conditions on nanophase ceramics $\left(\mathrm{Si}_{3} \mathrm{~N}_{4}, \mathrm{SiC}, \mathrm{TiO}_{2}\right.$, and $\mathrm{Al}_{2} \mathrm{O}_{3}$ ), and on silicates, aluminosilicates, and zeolites. 


\section{RESEARCH ACCOMPLISHMENTS}

This report summarizes our research accomplishments in materials simulations and high performance computing during the first year of DOE funding. The work reported here was carried out on parallel architectures (an 8,192-node MasPar 1208B, an 8-node Intel iPSC/860) system, a 64cell iWarp machine, and a Silicon Graphics Power Center 4D/380VGX) in our Concurrent Computing Laboratory for Materials Simulations (CCLMS). (Figure 1 shows schematically the set up of CCLMS.) Multi-million particle atomistic simulations are being carried out on the 512-node Touchstone Delta machine. The next phase of this project will also utilize a 56-node Intel Paragon system which is being acquired with our latest infrastructure enhancement grant from the State of Louisiana.

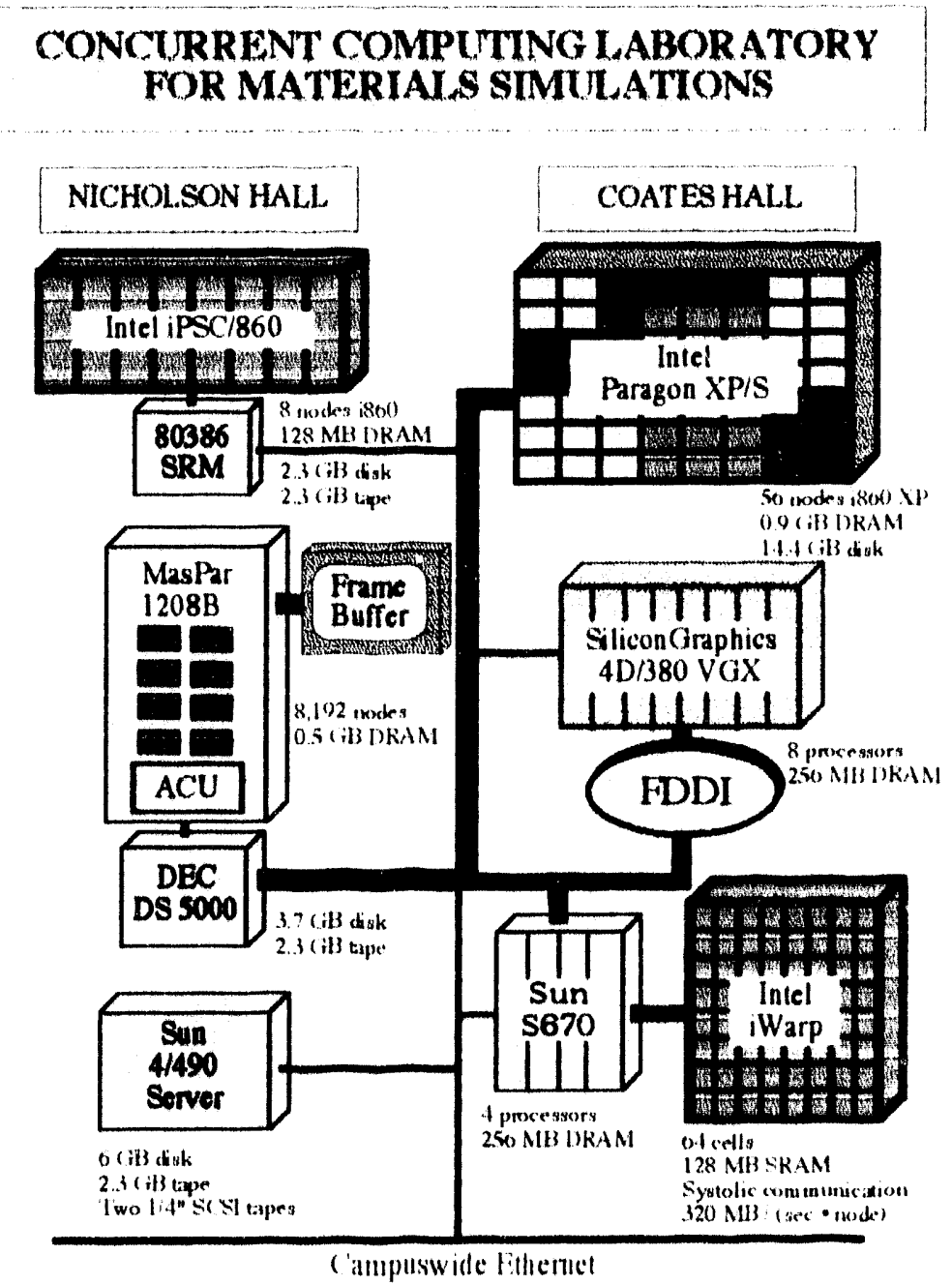

Figure 1: Computing facilities in the CCLMS' two parallel computing laboratories. 


\section{$\$ 1$ Multi-million Particle Molecular-dynamics Simulations on PARALlel MACHINES}

A variety of technologically important materials - for example, cluster-assembled nanophase materials, porous glasses, amorphous polymers - are composed of large-scale structural units, each consisting of $10^{3}$ to $10^{5}$ atoms which interact via long-range Coulomb and three-body covalent potentials. Condensed-phase simulations of these materials require large system sizes exceeding $100^{6}$ particles.

Highly efficient algorithms have been developed to carry out these "Grand Challenge" materials simulations on parallel computers. The algorithms employ the divide-and-conquer strategy shown in Fig. 2 to reduce computations by several orders of magnitude. The long-range Coulomb interaction experienced by a particle is computed by partitioning it into two parts: 1) a near-field contribution due to direct interaction with particles in neighboring boxes; and 2) a far-field contribution due to distant charged regions. The near-field contribution can be computed efficiently with the linked-list scheme. The far-field contribution is calculated with recursive domain decomposition of the system and a truncated multipole expansion for interactions between distant charged regions. The multipole expansion of the potential due to charges in each subdomain are combined hierarchicilly in such a way that only $\mathrm{O}(\mathrm{N})$ operations are required.

For short-ranged two-body and three-body interactions, we have employed a multiple-time-step scheme (MTS) scheme in which the force on a particle is subdivided into primary, secondary, and tertiary components (see Fig. 2 (c)). A significant reduction in computation is achieved by exploiting different time scales of these force components. The primary forces arise from nearest neighbors and they are updated after every MD time step. Compared to primary interactions, the secondary and tertiary forces vary slowly and therefore they are updated after $n_{1}(\sim 1.5)$ and $n_{2}(\sim 12())$ time steps, respectively. Between updates, the secondary and tertiary forces are calculated from Taylor's series.

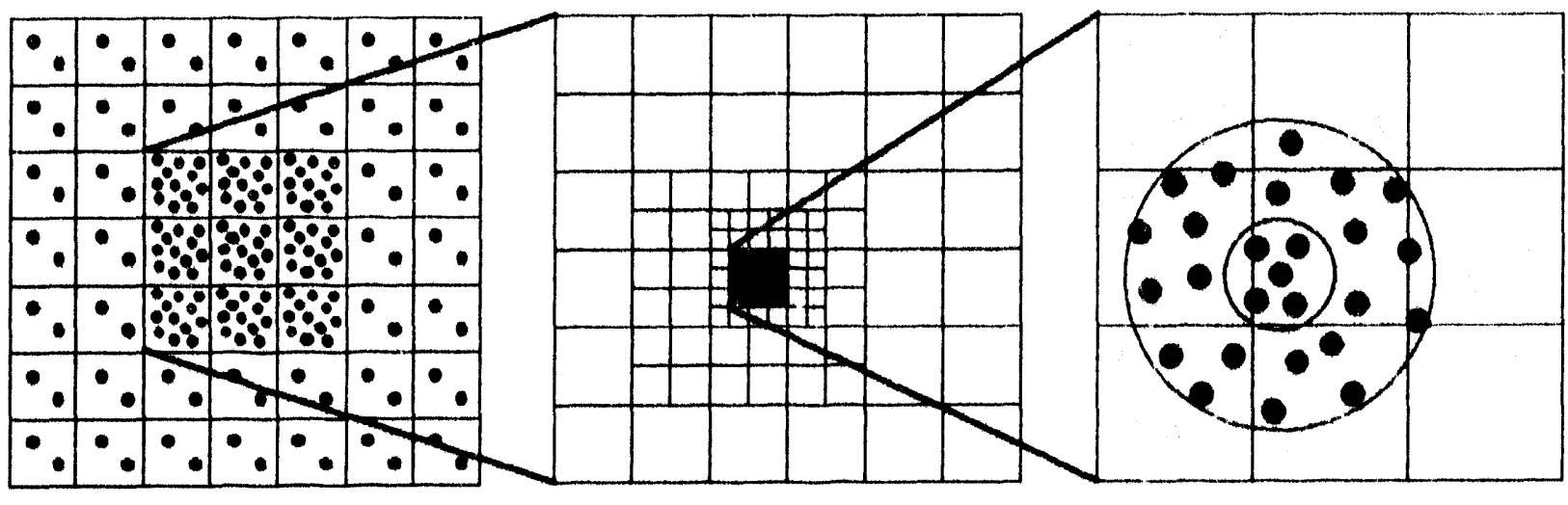

(a)

(b)

(c)

Figure 2: $\quad$ Multi-resolution in space. (a) Periodically repeated images of the original MD box. Replacing far images by a small number of particles with the same multipole expansion up to a certain order reduces the computation enormously while maintaining the necessary accuracy. (b) A hierarchy of cells used in the fast multipole method. (c) The near-field force on a particle (red) is due to primary (blue), secondary (green), and tertiary (gray) neighbor particles. 
Figure 3 shows the overall performance of the multi-resolution MTS-MD approach on the 512node Touchstone Delta machine. For a 4.2 million-particle silica glass, a single MD step takes only 4.84 seconds! The execution time scales linearly with the size of the system and the computation dominates the communication time. This is the most efficient implementation of the MD approach.

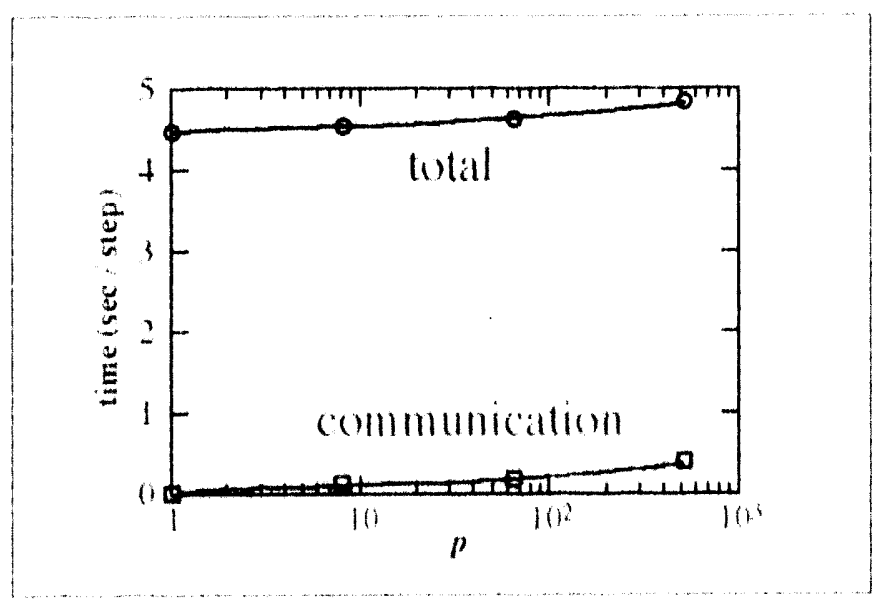

Figure 3: Execution time (circles) and communication time (squares) per MD time step for SiO) Here $p$ is the number of nodes of the Touchstone Delta machine. The size of the system, $N$, increases as $8232 \mathbf{p}$. Because of excellent scalability of multi-resolution algorithms, the execution and communication times remain almost constant with $\mathbf{p}$ and $\mathrm{N}$.

\section{$\$ 2$ StruCtural Transformation in MICroporols SII.JCA}

Aerogel silica, a porous form of $\mathrm{SiO}_{2}$ prepared by hypercritical drying of an alcoholic silica gel, is an environmentally safe material with a large thermal resistance which makes it a suitable alternative to chlorofluorocarbon (CFC)-foamed plastic in thermal insulation of commercial and houschold refrigerators. Scientists at Lawrence Livermore National Laboratory and Lawrence Berkeley Laboratory are investigating various other commercial uses of aerogel silica. These include passive solar energy collection devices, catalysis and chemical separation, and optical switches where porous materials are used as embedding frameworks for quantum-confined semiconducting microclusters.

From the technological standpoint, it is important to know the size distribution, the internal surface area and surface-to-volume ratio, and the interface texture of pores. Molecular-dy'namics simulations were performed to determine these structural parameters over a wide range of densities, from $2.2 \mathrm{~g} / \mathrm{cm}^{3}$ to as low as $0.1 \mathrm{~g} / \mathrm{cm}^{3}$. Snapshots of atomic positions in Fig. 4 reveal a structural transition from the condensed amorphous phase to a low-density porous phase. The latter is a fractal network whose dimensionality decreases rapidly with a decrease in the density. The density dependence of the internal surface area is in good agreement with X-ray measurements. 


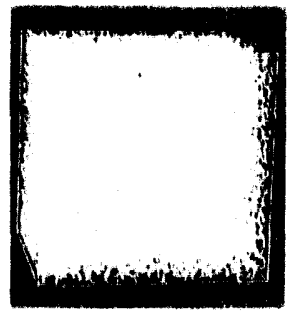

(a)

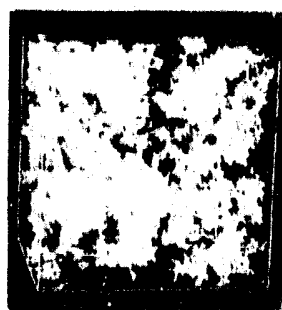

(c)

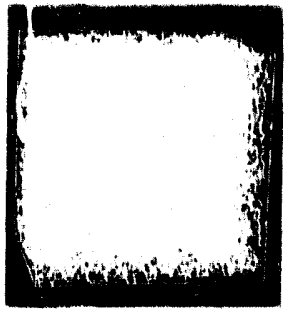

(b)

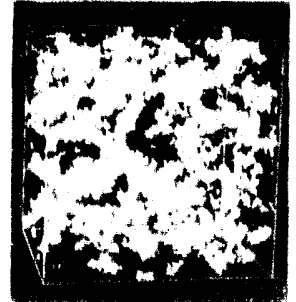

(t)

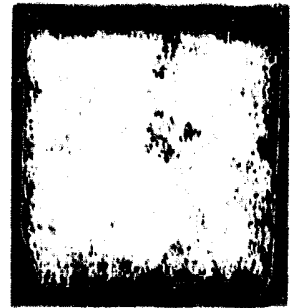

(c)

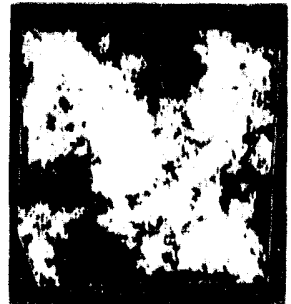

(g)

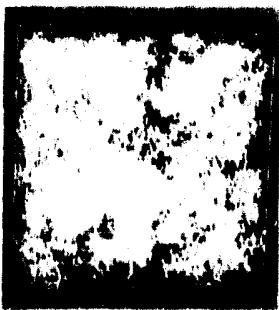

(d)

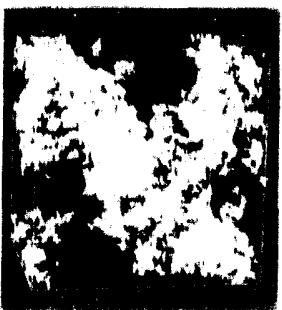

(h)

Figure 4: Snapshots of MD configurations showing $\mathrm{Si}$ O bonds in room-temperature porous $\mathrm{Si}()_{2}$ glasses at densities (a) 2.2 , (b) $1.6,($ (c) $)\left(0.8\right.$, (d) 0.4 , (c) 0.2 , and (f) $0.1 \mathrm{~g} / \mathrm{cm}^{3}$. Hore (g) and (h) are smapshots of systems with densities 0.2 and at $\left(0.1 \mathrm{~g} / \mathrm{cm}^{3}\right.$ at $1,(0)(0) \mathrm{K}$.

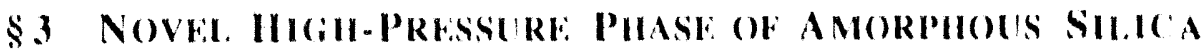

Recent in-situ high pressure $X$-ray measurements at the National Symchrotron light Source at Brookhaven National I aboratory revealed dramatic shanges in the structure of amorphous silical. Molecular dynamics simulations were performed to gain insight into the microscopies of structural changes at high pressutes. Under normal conditions, amorphous silicat is a disordered network of

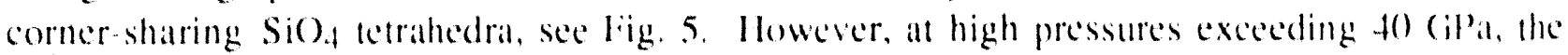
simulations reveal a new disordered network consisting of comer-sharing and edge bonded SiOh octahedra, sec ligh 5
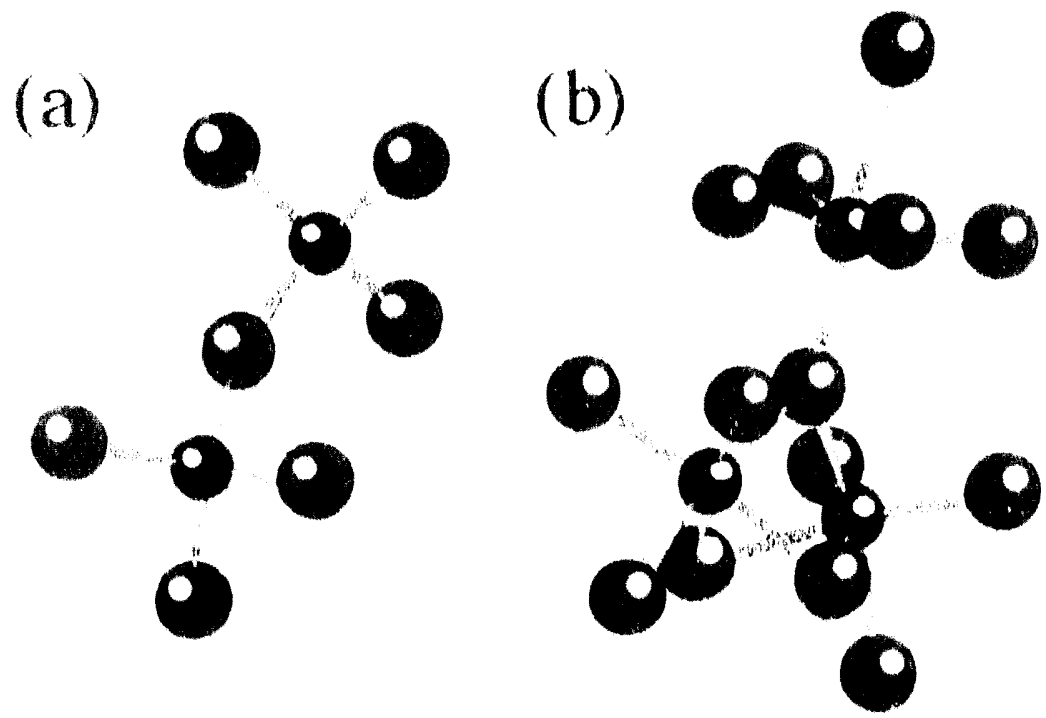

Figure 5: a) Corner-sharing $\mathrm{Si}()_{4}$ letrahedra in silica glass an normal density, $2.2 \mathrm{~g} / \mathrm{cm}^{3}$; and b) corner-sharing and edge bonded $\mathrm{SiO}_{6}$ octahedra in the new high pressure phase of silica all aldensity of $4.28 \mathrm{~g} / \mathrm{cm}^{3}$. 


\section{$\$ 4$ S'Tructural and DYNamical CORRELations in ORIENTATIONALLY DisORDERED SOLID C60}

Since the breakthrough in the synthesis of $\mathrm{C}_{6(0)}$ and the discovery of superconductivity in $\mathrm{K}_{3} \mathrm{C}_{60}$ and $\mathrm{Rb}_{3} \mathrm{C}_{60}$, there has been considerable interest in the chemical and physical properties of these molecular forms of carbon. Using the tight-binding-molecular-dynamics method, we have investigated inter- and intra-molecular phonon dispersions and densities of states (DOS) of orientationally ordered and disordered solid $\mathrm{C}_{60}$. Both inter-molecular and intra-molecular phonons show strong dispersions. Inter-molecular phonon DOS extends up to $7.6 \mathrm{meV}$ and shows libron peaks at $2.3 \mathrm{meV}$ and $3.7 \mathrm{meV}$. Orientational disorder softens libron modes. Intra-molecular phonons below $70 \mathrm{meV}$ also show significant dispersion. The calculated phonon spectra agree very well with recent inelastic neutron scattering experiments. Under pressure, the orientational potential stiffens causing the libron frequency to increase at a rate of $0.4 \mathrm{~cm}^{-1} / \mathrm{kbar}$. Most Raman and infra-red active modes show strong dependence on pressure that are in good agreement with experiments.

Recently a new form of carbon, called graphitic tubule, has been discovered. Owing to the crystalline perfection, one dimensional nature, and various possible helical structures, graphitic tubules are expected to exhibit unusual mechanical, electronic, and optical properties. Realistic simulations of graphitic tubules require large system sizes which are tractable only with the tightbinding molecular dynamics method implemented on parallel supercomputers. Using this approach on the Touchstone Delta machine, we are investigating the relationship between structural and mechanical properties of graphitic tubules.

\section{$\$ 5$ Parallel Quantum Dynamics Simulations}

Quantum dynamics (QD) is the sole method available for simulating the real-time dynamics of electrons and ions. The core computational kernels of the QD approach are the time-dependent KohnSham equation in the density-functional formalism and Poisson's equation for the long-range electron-electron interaction. Parallel algorithms have been developed to implement these kernels on SIMD machines. The Kohn-Sham equation is solved by reducing large tridiagonal matrices to direct sums of $2 \times 2$ matrices which can be handled analytically. The solution to Poisson's equation is obtained by the dynamical-simulated-annealing approach. Both algorithms are scalable and require only nearest-neighbor communications. On MasPar's 8,192-node machine, the solution to KohnSham and Poisson's equations achieve 64\% (479 Mflops) and 61\% (460) Mflops) of the theoretical peak performance, respectively. The $\mathrm{QD}$ approach has been used to investigate electron transport in disordered materials.

\section{$\$ 6$ WORK IN PROGRESS}

\section{Structural, Dynamical, and Mechanical Properties of Nanophase Materials}

Nanocrystalline metals and ceramics and nanoglasses with unique electrical, magnetic, and mechanical properties have been synthesized by in-situ consolidation of nanometer size atom clusters under high vacuum conditions. We are investigating the relationship between the microscopic 
structure, atomic diffusion, and mechanical behavior as influenced by environment and extreme physical conditions of high temperatures, pressures, and loading rates in nanostructured $\mathrm{Si}_{3} \mathrm{~N}_{4}, \mathrm{SiC}_{\text {, }}$ $\mathrm{TiO}_{2}$, and $\mathrm{Al}_{2} \mathrm{O}_{3}$. Considering the technological importance of nanophase ceramics and the enormity of these atomistic simulations, this is indeed a "Grand Challenge" project.

\section{Fracture of Porous Glasses}

From the technological standpoint, fracture is one of the most important material-specific issues concerning porous materials. Molecular-dynamics simulations are being performed on the Touchstone Delta machine to investigate fracture toughness, dynamics of crack propagation, and critical exponents for the width of a crack as a function of its length in porous materials. Nanoindentor simulations will be performed to study ductility and plastic deformation. Local internal stresses and hot spots, which are responsible for mechanical failure, will be investigated.

\section{Simulations of Silicates, Aluminosilicates, and Zeolites}

We are developing a new simulation approach which includes dynamic charge transfer resulting from changes in the local environment. With this technique, structural and dynamical correlations and thermodynamic and mechanical properties of silicates and aluminosilicates will be investigated. Simulations will also be performed to determine equilibrium interparticle separations, bond-angle distributions, the size distribution of pores, phonon densities-of-states, and mechanical properties of zeolites as a function of $\mathrm{Si} / \mathrm{Al}$ ratio. Diffusion mechanisms for atoms and molecules in zeolite networks will be investigated. 
$\$ 7$ DOE SUpported PUblications, Invited Talks at Major Conferences, AND A BSTRACTS

\section{Publications (1992-1993)}

1. Structure of Rings in Vitreous $\mathrm{SiO}_{2}$

J. P. Rino, I. Ebbsjö, R. K. Kalia, A. Nakano, and P. Vashishta,

Phys. Rev. B 47, 3053 (1993).

2. Structural Correlations in Porous Silica: Molecular Dynamics Simulation on a Parallel Computer

A. Nakano, L. Bi, R. K. Kalia, and P. Vashishta,

Phys. Rev. Letters 71, 85 (1993).

3. Structural Transformation, Medium Range Order and Dynamical Behavior of $\mathrm{SiO}_{2}$ Glass at High Pressures

W. Jin, R. K. Kalia, P. Vashishta, and J. P. Rino,

Phys. Rev. Letters, in press.

4. Phonon dispersion and density of states of solid $\mathrm{C}_{60}$

J. Yu, R. K. Kalia, and P. Vashishta,

Applied Physics Letters, in press.

5. Computer Simulation of Network Glasses and Molecular Dynamics Algorithm on SIMD and MIMD Machines

P. Vashishta, D. L. Greenwell, R. K. Kalia, and A. Nakano,

in Recent Progress in Many-Body Theories, Vol. 3, eds. T. L. Ainworth, C. E.

Campbell, and B. E. Clements, E. Krotsceck (Plenum, New York, 1992), p. 481.

6. Achievements in Solid State Physics and Density Functional Theory

M. H. Kalos, and P. Vashishta,

in Recent Progress in Many-Body Theories, Vol. 3, eds. T. L. Ainworth, C. E.

Campbell, and B. E. Clements, E. Krotsceck (Plenum, New York, 1992), p. 493.

7. Parallel Multiple-Time Step-Molecular Dynamics with Three-Body Interaction

A. Nakano, P. Vashishta, and R. K. Kalia,

Computer Physics Communications, in press.

8. Structural and Dynamical Correlations in Glasses

W. Jin, J. P. Rino, P. Vashishta, R. K. Kalia, and A. Nakano, in Strongly Coupled Plasma Physics, edited by H. M. Van Horn and S. Ichirmaru (University of Rochester Press, Rochester, 1993), in press.

9. Molecular Dynamics Simulation of Network Glasses and Algorithms on Parallel (SIMD \& MIMD) Architectures

P. Vashishta, R. K. Kalia, W. Jin, A. Nakano, and D. L. Greenwell, in Computer Aided Innovation of New Materials Il, edited by M. Doyama et all. (North Holland, Amsterdam, 1993), p. 235.

10. Atomistic Simulations on Parallel Architectures

R. K. Kalia, W. Jin, S. de Leeuw, A. Nakano, and P. Vashishta, Int. J. Quantum Chemistry, in press. 
11. Dynamical Structure Factor and Vibrational Nomal Modes of $\mathrm{SiO}_{2}$ Glass W. Jin, R. K. Kalia, and P. Vashishta, Mat. Res. Soc. Symp. Proc. 291, 343 (1993).

12. Dynamical Structure Factor and Vibrational Properties of $\mathrm{SiO}_{2}$ Glass W. Jin, R. K. Kalia, P. Vashishta, and J. P. Rino, Phys. Rev. B, in press.

13. Classical and Quantum Simulations for Large Systems on Parallel Computers P. Vashishta, R. K. Kalia, and J. Yu, Mat. Res. Soc. Symp. Proc. 291, 3 (1993).

14. Structure, Fragmentation, and Phonons in Silicon Microclusters Wei Li, Rajiv K. Kalia, and Priya Vashishta, Mat. Res. Soc. Symp. Proc. 293, (1993).

\section{Invited Talks at Major Conferences (1992-1993)}

1. Structural and Dynamical Correlations in Cilasses

"International Conference on Physics of Strongly Coupled Plasmas",

Rochester, New York, August 17-21, 1992.

2. Molecular Dynamics Simulation of Strongly Covalent Systems with Coulomb Interactions "International Conference on Computational Physics for Condensed Matter Phenomena", Tokyo, Japan, September 19-21, 1992.

3. Molecular Dynamics Simulation of Network Glasses \& MD Algorithm on Parallel Computers "Second International Conference on Computer Applications to Materials and Molecular Science and Engineering", Yokohama, Japan, September 22-25, 1992.

4. Covalent Glasses at Large Positive and Negative Pressures - A Molecular Dynamicis Study "New Developments of Computer Based Design of Functional materials".

Osaka, Japan, September 29-30, 1992.

5. Molecular Dynamics Simulation of Covalent Glasses

"Southeastern Section Section American Physical Society Meeting", Oak Ridge, Tennessee, November 12-14, 1992.

6. Classical and Quantum Simulations for Large Systemus on Parallel Computers

"Theory and Modeling" Symposium at the Annual Materials Research Society Meeting, Boston, November 29-December 4, 1992.

7. Structure and Dynamics of Network Glasises at Very large Positive and Negative Pressures. A Molecular Dynamics Study

"Solid State Ionics" Symposium at the Annual Materials Research Society

Meeting, Boston, November 29-December 4, 1992.

8. Computational approach to nanotechnology on parallel architectures

"Mardi Gras '93, Concurrent Computing Conference in the Physical Sciences", Baton Rouge, Louisiana, February 18-20, 1993.

9. Covalent Glasses at Very Large Positive and Negative Pressures - A Molecular Dynamics Study

"The Metallurgical Society of America", Denver, Colorado, February 21-25, 1993. 
10. Structure and Dynamics of $\mathrm{SiO}_{2}$ at Very Large Positive and Negative Pressures

"American Chemical Society Meeting", Denver, Colorado, March 28-April 2, 1993.

11. Molecular Dynamics of Silica Under Very Large Positive and Negative Pressures on Parallel Computers

"High Performance Computing - 1993 Simulation MultiConference" .

Washington DC, March 29-April 1, 1993.

\section{Conference orpanizers provided most of the travel and other expenses.}

\section{Conference Presentations and Abstracts (1992-1993)}

1. Structural and Dynamical Correlations in Glasses

W. Jin, J. P. Rino, P. Vashishta, R. K. Kalia, and A. Nakano,

International Conference on the Physics of Strongly Coupled Plasmas, Rochester,

New York, August 17-21, 1992.

2. Dynamical Structure Factor and Vibrational Normal Modes of $\mathrm{SiO}_{2}$ Glass

W. Jin, S. de Leeuw, R. K. Kalia, and P. Vashishta,

MRS Fall 92 Abstracts, p. 490).

3. Structural and Dynamical Correlations in Stishovite and High Density Vitreous Silicit

W. Jin, R. K. Kalia, and P. Vashishta,

MRS Fall 92 Alstracts, p. 58.5.

4. Classical and Quantum Simulations for Large Systems on Parallel Computers

P. Vashishta, R. K. Kalia, and J. Yu,

MRS Fall 92 Abstracts, p. 475.

5. Structure, Fragmentation, and Phonons in Silicon Microclusters

Wei Li, Rajiv K. Kalia, and Priya Vashishti,

MRS Fall 92 Abstracts, p. 593.

6. Structure and Dynamics of Network Glasses at Very large Positive and Negative Pressures A Molecular Dynamics Study P. Vashishta and R. K. Kalia, MRS Fall 92 Abstracts, p. 584.

7. Molecular Dynamics Simulation of Aerogel Silica on Parallel Computers

A. Nakano, R. K. Kalia, and P. Vashishta, MRS Fall 92 Abstracts, p. 58.5.

8. Covalent Glasses at Large Positive and Negative Pressures: A Molecular Dynamics Study P. Vashishta, R. K. Kalia, A. Nakano, and W. Jin, 1993 Minerals, Metals and Materials Society Annual Meeting, Denver, CO, February $21-25,1993$, Abstracts, p. B35.

9. Structure and Dynamics of $\mathrm{SiO}_{2}$ at Very Large Positive and Negative Pressures P. Vashishta, W. Jin, R. K. Kalia, and A. Nakano, 1993 American Chemical Society National Meeting, Denver, CO, March 28

- April 2, 1993, Program, p. 95. 
10. Molecular Dynamics of Silica Under Very Large Positive and Negative Pressures on Parallel Computers

P. Vashishta, R. Kalia, W. Jin, and A. Nakano, 1993 Simulation MultiConference, Washington D.C., March 29 - April 1, 1993.

11. Dynamical Structure Factor and Vibrational Excitations of $\mathrm{SiO}_{2}$ Glass

W. Jin, R. K. Kalia, and P. Vashishta,

Bull. Am. Phys. Soc. 38, 574 (1993).

12. Structural Transformation, Intermediate-Range Order and Dynamical Behavior of $\mathrm{SiO}_{2} \mathrm{Gläss}$ at High Pressures

W. Jin, R. K. Kalia, and P. Vashishta,

Bull. Am. Phys. Soc. 38, 675 (1993).

13. Structural Correlations in Porous Silica: Parallel Molecular Dynamics Simulations

A. Nakano, R. K. Kalia, and P. Vashishta,

Bul!. Am. Phys. Soc. 38, 673 (1993).

14. Phonon Dispersion and Density of States of Solid $\mathrm{C}_{60}$

J. Yu, R. K. Kalia, and P. Vashishta,

Bull. Am. Phys. Soc. 38, 441 (1993). 
(This page is intentionally left blank.) 

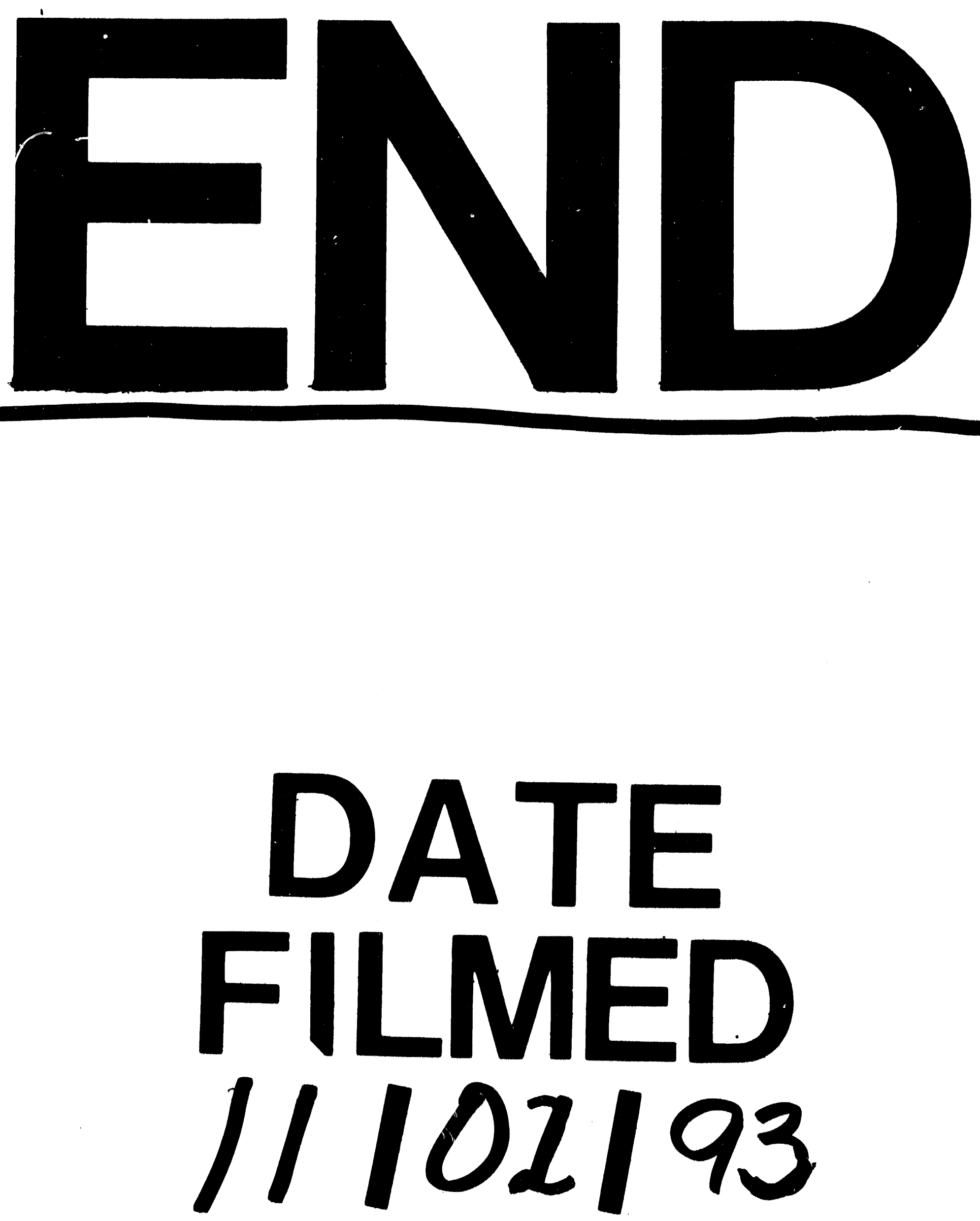
